\title{
Some fixed point theorems in complex valued metric spaces
}

\author{
Kittipong Sitthikul and Satit Saejung*
}

\section{"Correspondence:} saejung@kku.ac.th

Department of Mathematics, Khon Kaen University, Khon Kaen, 40002, Thailand

\begin{abstract}
Owning the concept of complex valued metric spaces introduced by Azam et al., we prove several fixed point theorems for mappings satisfying certain point-dependent contractive conditions. The main results announced by Sintunavarat and Kumam (J. Inequal. Appl. 2012:84, 2012), Rouzkard and Imdad (Comput. Math. Appl., 2012, doi:10.1016/j.camwa.2012.02.063), and Dass and Gupta (Indian J. Pure Appl. Math. 6(12):1455-1458, 1975) are deduced from our results under weaker assumptions.
\end{abstract}

\section{Introduction}

The concept of a complex valued metric space which is a generalization of the classical metric space was recently introduced by Azam, Fisher and Khan (see [1]). To mention this, let us recall a natural relation $\precsim$ on $\mathbb{C}$, the set of complex numbers as follows: for $z_{1}, z_{2} \in \mathbb{C}$

$$
\begin{aligned}
& z_{1} \precsim z_{2} \stackrel{\text { def }}{\Longleftrightarrow} \operatorname{Re}\left(z_{1}\right) \leq \operatorname{Re}\left(z_{2}\right) \quad \text { and } \quad \operatorname{Im}\left(z_{1}\right) \leq \operatorname{Im}\left(z_{2}\right), \\
& z_{1} \prec z_{2} \stackrel{\text { def }}{\Longleftrightarrow} \operatorname{Re}\left(z_{1}\right)<\operatorname{Re}\left(z_{2}\right) \quad \text { and } \quad \operatorname{Im}\left(z_{1}\right)<\operatorname{Im}\left(z_{2}\right) .
\end{aligned}
$$

Definition 1.1 Let $X$ be a nonempty set. A mapping $d: X \times X \rightarrow \mathbb{C}$ is called a complex valued metric on $X$ if the following conditions are satisfied:

(CM1) $0 \precsim d(x, y)$ for all $x, y \in X$ and $d(x, y)=0 \Longleftrightarrow x=y$;

(CM2) $d(x, y)=d(y, x)$ for all $x, y \in X$;

(CM3) $d(x, y) \precsim d(x, z)+d(z, y)$ for all $x, y, z \in X$.

In this case, we say that $(X, d)$ is a complex valued metric space.

It is obvious that this concept is a generalization of the classical metric. In fact, if $d$ : $X \times X \rightarrow \mathbb{R}$ satisfies (CM1)-(CM3), then this $d$ is a metric in the classical sense; that is, the following conditions are satisfied:

(M1) $0 \leq d(x, y)$ for all $x, y \in X$ and $d(x, y)=0 \Longleftrightarrow x=y$;

(M2) $d(x, y)=d(y, x)$ for all $x, y \in X$;

(M3) $d(x, y) \leq d(x, z)+d(z, y)$ for all $x, y, z \in X$.

The following definition is an analogue of several concepts in the classical theory of metric spaces and they are discussed in [1]. There are also other interesting types of generalization of metric spaces; for example, see $[2,3]$.

Definition 1.2 Suppose that $(X, d)$ is a complex valued metric space. 
- We say that a sequence $\left\{x_{n}\right\}$ is a Cauchy sequence if for every $0 \prec c \in \mathbb{C}$ there exists an integer $N$ such that $d\left(x_{n}, x_{m}\right) \prec c$ for all $n, m \geq N$.

- We say that $\left\{x_{n}\right\}$ converges to an element $x \in X$ if for every $0 \prec c \in \mathbb{C}$ there exists an integer $N$ such that $d\left(x_{n}, x\right) \prec c$ for all $n \geq N$. In this case, we write $x_{n} \stackrel{d}{\rightarrow} x$.

- We say that $(X, d)$ is complete if every Cauchy sequence in $X$ converges to a point in $X$.

The following fact is summarized from Azam, Fisher and Khan's paper [1]. In fact, (b) and (c) of Proposition 1.3 are their Lemmas 2 and 3.

Proposition 1.3 Let $(X, d)$ be a complex value metric space. Suppose that $d=d_{1}+i d_{2}$ where $d_{1}, d_{2}: X \times X \rightarrow \mathbb{R}$, that is, $d_{1}=\operatorname{Re}(d)$ and $d_{2}=\operatorname{Im}(d)$. Then the following assertions hold.

(a) $|d|=\left(d_{1}^{2}+d_{2}^{2}\right)^{1 / 2}: X \times X \rightarrow \mathbb{R}$ is a (classical) metric on $X$.

(b) If $\left\{x_{n}\right\}$ is a sequence in $X$ and $x \in X$, then $x_{n} \stackrel{d}{\rightarrow} x$ if and only if $x_{n} \stackrel{|d|}{\rightarrow} x$.

(c) $(X, d)$ is complete if and only if $(X,|d|)$ is complete.

The following common fixed point theorem was also proved by Azam, Fisher and Khan. This can be viewed as a generalization of the well-known Banach fixed point theorem.

Theorem 1.4 ([1]) Let $(X, d)$ be a complete complex valued metric space, and let $\lambda, \mu$ be nonnegative real numbers such that $\lambda+\mu<1$. Suppose that $S, T: X \rightarrow X$ are mappings satisfying:

$$
d(S x, T y) \precsim \lambda d(x, y)+\frac{\mu d(x, S x) d(y, T y)}{1+d(x, y)} \quad \forall x, y \in X .
$$

Then $S$ and $T$ have a unique common fixed point.

In this paper, we continue the study of fixed point theorems in complex valued metric spaces. The obtained results are generalizations of recent results proved by Sintunavarat and Kumam [4], Rouzkard and Imdad [5]. Moreover, we improve several assumptions on the involved mappings. It should be noted that there are also some different fixed point theorems recently proved in [6].

\section{Main result}

Throughout the paper, let $(X, d)$ be a complete complex valued metric space and $S, T$ : $X \rightarrow X$.

Proposition 2.1 Let $x_{0} \in X$ and define the sequence $\left\{x_{n}\right\}$ by

$$
\begin{aligned}
& x_{2 n+1}=S x_{2 n}, \\
& x_{2 n+2}=T x_{2 n+1}, \quad \text { for all } n=0,1,2, \ldots .
\end{aligned}
$$

Assume that there exists a mapping $\lambda: X \times X \rightarrow[0,1)$ satisfying

$$
\lambda(T S x, y) \leq \lambda(x, y) \quad \text { and } \quad \lambda(x, S T y) \leq \lambda(x, y) \quad \text { for all } x, y \in X .
$$

Then $\lambda\left(x_{2 n}, y\right) \leq \lambda\left(x_{0}, y\right)$ and $\lambda\left(x, x_{2 n+1}\right) \leq \lambda\left(x, x_{1}\right)$ for all $x, y \in X$ and $n=0,1,2, \ldots$ 
Proof Let $x, y \in X$ and $n=0,1,2, \ldots$. Then we have

$$
\lambda\left(x_{2 n}, y\right)=\lambda\left(T S x_{2 n-2}, y\right) \leq \lambda\left(x_{2 n-2}, y\right)=\lambda\left(T S x_{2 n-4}, y\right) \leq \cdots \leq \lambda\left(x_{0}, y\right) .
$$

Similarly, we have

$$
\lambda\left(x, x_{2 n+1}\right)=\lambda\left(x, S T x_{2 n-1}\right) \leq \lambda\left(x, x_{2 n-1}\right)=\lambda\left(x, S T x_{2 n-3}\right) \leq \cdots \leq \lambda\left(x, x_{1}\right) .
$$

Lemma 2.2 Let $\lambda, \mu: X \times X \rightarrow[0,1)$ and $x, y \in X$. If $S$ and $T$ satisfy

$$
\begin{aligned}
& d(S x, T S x) \precsim \lambda(x, S x) d(x, S x)+\mu(x, S x) \frac{d(x, S x) d(S x, T S x)}{1+d(x, S x)}, \\
& d(S T y, T y) \precsim \lambda(T y, y) d(T y, y)+\mu(T y, y) \frac{d(T y, S T y) d(y, T y)}{1+d(T y, y)},
\end{aligned}
$$

then

$$
\begin{aligned}
& |d(S x, T S x)| \leq \lambda(x, S x)|d(x, S x)|+\mu(x, S x)|d(S x, T S x)|, \\
& |d(S T y, T y)| \leq \lambda(T y, y)|d(T y, y)|+\mu(T y, y)|d(T y, S T y)|,
\end{aligned}
$$

respectively.

Proof We can write

$$
\begin{aligned}
|d(S x, T S x)| & \leq\left|\lambda(x, S x) d(x, S x)+\mu(x, S x) \frac{d(x, S x) d(S x, T S x)}{1+d(x, S x)}\right| \\
& \leq \lambda(x, S x)|d(x, S x)|+\mu(x, S x)\left|\frac{d(x, S x)}{1+d(x, S x)}\right||d(S x, T S x)| \\
& \leq \lambda(x, S x)|d(x, S x)|+\mu(x, S x)|d(S x, T S x)| .
\end{aligned}
$$

Similarly, we get

$$
\begin{aligned}
|d(S T y, T y)| & \leq\left|\lambda(T y, y) d(T y, y)+\mu(T y, y) \frac{d(T y, S T y) d(y, T y)}{1+d(T y, y)}\right| \\
& \leq \lambda(T y, y)|d(y, T y)|+\mu(T y, y)\left|\frac{d(y, T y)}{1+d(y, T y)}\right||d(T y, S T y)| \\
& \leq \lambda(T y, y)|d(y, T y)|+\mu(T y, y)|d(S T y, T y)| .
\end{aligned}
$$

Lemma 2.3 Let $\left\{x_{n}\right\}$ be a sequence in $X$ and $h \in[0,1)$. If $a_{n}=\left|d\left(x_{n}, x_{n+1}\right)\right|$ satisfies

$$
a_{n} \leq h a_{n-1}, \quad \text { for all } n \in \mathbb{N}
$$

then $\left\{x_{n}\right\}$ is a Cauchy sequence.

Proof Let $h \in[0,1)$. Then

$$
a_{n} \leq h a_{n-1} \leq h^{2} a_{n-2} \leq \cdots \leq h^{n} a_{0}, \quad \text { for all } n \in \mathbb{N} .
$$


For $m, n \in \mathbb{N}$ such that $m>n$, we have

$$
\begin{aligned}
\left|d\left(x_{n}, x_{m}\right)\right| & \leq a_{n}+a_{n+1}+\cdots+a_{m-1} \\
& \leq h^{n}\left(1+h+h^{2}+\cdots+h^{m-n-1}\right) a_{0} \\
& \leq \frac{h^{n}}{1-h} a_{0} .
\end{aligned}
$$

Thus, we have $\left|d\left(x_{n}, x_{m}\right)\right| \rightarrow 0$ as $n \rightarrow \infty$, and hence $\left\{x_{n}\right\}$ is a Cauchy sequence.

Theorem 2.4 Let $(X, d)$ be a complete complex valued metric space and $S, T: X \rightarrow X$. If there exist mappings $\lambda, \mu, \gamma: X \times X \rightarrow[0,1)$ such that for all $x, y \in X$ :

(a) $\lambda(T S x, y) \leq \lambda(x, y)$ and $\lambda(x, S T y) \leq \lambda(x, y)$,

$$
\begin{aligned}
& \mu(T S x, y) \leq \mu(x, y) \text { and } \mu(x, S T y) \leq \mu(x, y), \\
& \gamma(T S x, y) \leq \gamma(x, y) \text { and } \gamma(x, S T y) \leq \gamma(x, y) ;
\end{aligned}
$$

(b) $\lambda(x, y)+\mu(x, y)+\gamma(x, y)<1$;

(c)

$$
d(S x, T y) \precsim \lambda(x, y) d(x, y)+\mu(x, y) \frac{d(x, S x) d(y, T y)}{1+d(x, y)}+\gamma(x, y) \frac{d(y, S x) d(x, T y)}{1+d(x, y)} .
$$

Then $S$ and $T$ have a unique common fixed point.

Proof Let $x, y \in X$. From (6), we consider

$$
\begin{aligned}
d(S x, T S x) \precsim & \lambda(x, S x) d(x, S x)+\mu(x, S x) \frac{d(x, S x) d(S x, T S x)}{1+d(x, S x)} \\
& +\gamma(x, S x) \frac{d(S x, S x) d(x, T S x)}{1+d(x, S x)} \\
= & \lambda(x, S x) d(x, S x)+\mu(x, S x) \frac{d(x, S x) d(S x, T S x)}{1+d(x, S x)} .
\end{aligned}
$$

From Lemma 2.2, we have

$$
|d(S x, T S x)| \leq \lambda(x, S x)|d(x, S x)|+\mu(x, S x)|d(S x, T S x)| .
$$

Similarly, we get

$$
\begin{aligned}
d(S T y, T y) \precsim & \lambda(T y, y) d(T y, y)+\mu(T y, y) \frac{d(T y, S T y) d(y, T y)}{1+d(T y, y)} \\
& +\gamma(T y, y) \frac{d(y, S T y) d(T y, T y)}{1+d(T y, y)} \\
= & \lambda(T y, y) d(T y, y)+\mu(T y, y) \frac{d(T y, S T y) d(y, T y)}{1+d(T y, y)} .
\end{aligned}
$$

From Lemma 2.2, we have

$$
|d(S T y, T y)| \leq \lambda(T y, y)|d(T y, y)|+\mu(T y, y)|d(T y, S T y)| .
$$


Let $x_{0} \in X$ and the sequence $\left\{x_{n}\right\}$ be defined by (2). We show that $\left\{x_{n}\right\}$ is a Cauchy sequence. From Proposition 2.1, (7), (8) and for all $k=0,1,2, \ldots$, we have

$$
\begin{aligned}
\left|d\left(x_{2 k+1}, x_{2 k}\right)\right|= & \left|d\left(S T x_{2 k-1}, T x_{2 k-1}\right)\right| \\
\leq & \lambda\left(T x_{2 k-1}, x_{2 k-1}\right)\left|d\left(T x_{2 k-1}, x_{2 k-1}\right)\right| \\
& +\mu\left(T x_{2 k-1}, x_{2 k-1}\right)\left|d\left(T x_{2 k-1}, S T x_{2 k-1}\right)\right| \\
= & \lambda\left(x_{2 k}, x_{2 k-1}\right)\left|d\left(x_{2 k-1}, x_{2 k}\right)\right|+\mu\left(x_{2 k}, x_{2 k-1}\right)\left|d\left(x_{2 k}, x_{2 k+1}\right)\right| \\
\leq & \lambda\left(x_{0}, x_{2 k-1}\right)\left|d\left(x_{2 k-1}, x_{2 k}\right)\right|+\mu\left(x_{0}, x_{2 k-1}\right)\left|d\left(x_{2 k+1}, x_{2 k}\right)\right| \\
\leq & \lambda\left(x_{0}, x_{1}\right)\left|d\left(x_{2 k-1}, x_{2 k}\right)\right|+\mu\left(x_{0}, x_{1}\right)\left|d\left(x_{2 k+1}, x_{2 k}\right)\right|,
\end{aligned}
$$

which implies that

$$
\left|d\left(x_{2 k+1}, x_{2 k}\right)\right| \leq \frac{\lambda\left(x_{0}, x_{1}\right)}{1-\mu\left(x_{0}, x_{1}\right)}\left|d\left(x_{2 k-1}, x_{2 k}\right)\right| .
$$

Similarly, we get

$$
\begin{aligned}
\left|d\left(x_{2 k+2}, x_{2 k+1}\right)\right| & =\left|d\left(T S x_{2 k}, S x_{2 k}\right)\right| \\
& \leq \lambda\left(x_{2 k}, S x_{2 k}\right)\left|d\left(x_{2 k}, S x_{2 k}\right)\right|+\mu\left(x_{2 k}, S x_{2 k}\right)\left|d\left(S x_{2 k}, T S x_{2 k}\right)\right| \\
& =\lambda\left(x_{2 k}, x_{2 k+1}\right)\left|d\left(x_{2 k}, x_{2 k+1}\right)\right|+\mu\left(x_{2 k}, x_{2 k+1}\right)\left|d\left(x_{2 k+1}, x_{2 k+2}\right)\right| \\
& \leq \lambda\left(x_{0}, x_{2 k+1}\right)\left|d\left(x_{2 k}, x_{2 k+1}\right)\right|+\mu\left(x_{0}, x_{2 k+1}\right)\left|d\left(x_{2 k+2}, x_{2 k+1}\right)\right| \\
& \leq \lambda\left(x_{0}, x_{1}\right)\left|d\left(x_{2 k}, x_{2 k+1}\right)\right|+\mu\left(x_{0}, x_{1}\right)\left|d\left(x_{2 k+2}, x_{2 k+1}\right)\right|,
\end{aligned}
$$

which implies that

$$
\left|d\left(x_{2 k+2}, x_{2 k+1}\right)\right| \leq \frac{\lambda\left(x_{0}, x_{1}\right)}{1-\mu\left(x_{0}, x_{1}\right)}\left|d\left(x_{2 k}, x_{2 k+1}\right)\right| .
$$

Let $h=\frac{\lambda\left(x_{0}, x_{1}\right)}{1-\mu\left(x_{0}, x_{1}\right)}<1$. Then we have

$$
\left|d\left(x_{n+1}, x_{n}\right)\right| \leq h\left|d\left(x_{n-1}, x_{n}\right)\right|, \quad \text { for all } n \in \mathbb{N} .
$$

From Lemma 2.3, we have $\left\{x_{n}\right\}$ is a Cauchy sequence in $(X, d)$. By the completeness of $X$, there exists $z \in X$ such that $x_{n} \rightarrow z$ as $n \rightarrow \infty$.

Next, we show that $z$ is a fixed point of $S$. By (6) and Proposition 2.1, we have

$$
\begin{aligned}
d(z, S z) \precsim & d\left(z, T x_{2 n+1}\right)+d\left(T x_{2 n+1}, S z\right) \\
= & d\left(z, x_{2 n+2}\right)+d\left(S z, T x_{2 n+1}\right) \\
\precsim & d\left(z, x_{2 n+2}\right)+\lambda\left(z, x_{2 n+1}\right) d\left(z, x_{2 n+1}\right)+\mu\left(z, x_{2 n+1}\right) \frac{d(z, S z) d\left(x_{2 n+1}, T x_{2 n+1}\right)}{1+d\left(z, x_{2 n+1}\right)} \\
& +\gamma\left(z, x_{2 n+1}\right) \frac{d\left(x_{2 n+1}, S z\right) d\left(z, T x_{2 n+1}\right)}{1+d\left(z, x_{2 n+1}\right)}
\end{aligned}
$$




$$
\begin{aligned}
& \precsim d\left(z, x_{2 n+2}\right)+\lambda\left(z, x_{1}\right) d\left(z, x_{2 n+1}\right)+\mu\left(z, x_{1}\right) \frac{d(z, S z) d\left(x_{2 n+1}, x_{2 n+2}\right)}{1+d\left(z, x_{2 n+1}\right)} \\
& \quad+\gamma\left(z, x_{1}\right) \frac{d\left(x_{2 n+1}, S z\right) d\left(z, x_{2 n+2}\right)}{1+d\left(z, x_{2 n+1}\right)} .
\end{aligned}
$$

Thus, $d(z, S z)=0$ and hence $z=S z$.

We also show that $z$ is a fixed point of $T$. By (6), we have

$$
\begin{aligned}
d(z, T z) \precsim & d\left(z, S x_{2 n}\right)+d\left(S x_{2 n}, T z\right) \\
\precsim & d\left(z, x_{2 n+1}\right)+\lambda\left(x_{2 n}, z\right) d\left(x_{2 n}, z\right)+\mu\left(x_{2 n}, z\right) \frac{d\left(x_{2 n}, S x_{2 n}\right) d(z, T z)}{1+d\left(x_{2 n}, z\right)} \\
& +\gamma\left(x_{2 n}, z\right) \frac{d\left(z, S x_{2 n}\right) d\left(x_{2 n}, T y\right)}{1+d\left(x_{2 n}, z\right)} \\
\precsim & d\left(z, x_{2 n+1}\right)+\lambda\left(x_{0}, z\right) d\left(x_{2 n}, z\right)+\mu\left(x_{0}, z\right) \frac{d\left(x_{2 n}, x_{2 n+1}\right) d(z, T z)}{1+d\left(x_{2 n}, z\right)} \\
& +\gamma\left(x_{0}, z\right) \frac{d\left(z, x_{2 n+1}\right) d\left(x_{2 n}, T y\right)}{1+d\left(x_{2 n}, z\right)} .
\end{aligned}
$$

Thus, $d(z, T z)=0$ and hence $z=T z$. Therefore, $z$ is a common fixed point of $S$ and $T$.

Finally, we show the uniqueness. Suppose that there is $z^{\prime \prime} \in X$ such that $z^{\prime \prime}=S z^{\prime \prime}=T z^{\prime \prime}$.

Then

$$
\begin{aligned}
d\left(z, z^{*}\right) & =d\left(S z, T z^{*}\right) \\
& \precsim \lambda\left(z, z^{*}\right) d\left(z, z^{*}\right)+\mu\left(z, z^{*}\right) \frac{d(z, S z) d\left(z^{*}, T z^{*}\right)}{1+d\left(z, z^{*}\right)}+\gamma\left(z, z^{*}\right) \frac{d\left(z^{*}, S z\right) d\left(z, T z^{*}\right)}{1+d\left(z, z^{*}\right)} \\
& =\lambda\left(z, z^{*}\right) d\left(z, z^{*}\right)+\gamma\left(z, z^{*}\right) \frac{d\left(z^{*}, z\right) d\left(z, z^{*}\right)}{1+d\left(z, z^{*}\right)} .
\end{aligned}
$$

Therefore, we have

$$
\begin{aligned}
\left|d\left(z, z^{*}\right)\right| & \leq \lambda\left(z, z^{\prime \prime}\right)\left|d\left(z, z^{*}\right)\right|+\gamma\left(z, z^{*}\right)\left|d\left(z, z^{\prime \prime}\right)\right|\left|\frac{d\left(z, z^{*}\right)}{1+d\left(z, z^{*}\right)}\right| \\
& \leq \lambda\left(z, z^{*}\right)\left|d\left(z, z^{*}\right)\right|+\gamma\left(z, z^{*}\right)\left|d\left(z, z^{*}\right)\right| \\
& \leq\left(\lambda\left(z, z^{*}\right)+\gamma\left(z, z^{*}\right)\right)\left|d\left(z, z^{\prime \prime}\right)\right| .
\end{aligned}
$$

Since $\lambda\left(z, z^{*}\right)+\gamma\left(z, z^{*}\right)<1$, we have $\left|d\left(z, z^{*}\right)\right|=0$. Thus $z=z^{*}$.

By setting $S=T$ in Theorem 2.4, we deduce the following corollary.

Corollary 2.5 Let $(X, d)$ be a complete complex valued metric space and $T: X \rightarrow X$. If there exist mappings $\lambda, \mu, \gamma: X \times X \rightarrow[0,1)$ such that for all $x, y \in X$ :

(a) $\lambda(T x, y) \leq \lambda(x, y)$ and $\lambda(x, T y) \leq \lambda(x, y)$, $\mu(T x, y) \leq \mu(x, y)$ and $\mu(x, T y) \leq \mu(x, y)$, $\gamma(T x, y) \leq \gamma(x, y)$ and $\gamma(x, T y) \leq \gamma(x, y)$

(b) $\lambda(x, y)+\mu(x, y)+\gamma(x, y)<1$; 
(c)

$$
d(T x, T y) \precsim \lambda(x, y) d(x, y)+\mu(x, y) \frac{d(x, T x) d(y, T y)}{1+d(x, y)}+\gamma(x, y) \frac{d(y, T x) d(x, T y)}{1+d(x, y)} .
$$

Then $T$ has a unique common fixed point.

By choosing $\gamma=0$ in Theorem 2.4, we deduce the following corollary.

Corollary 2.6 Let $(X, d)$ be a complete complex valued metric space and $S, T: X \rightarrow X$. If there exist mappings $\lambda, \mu: X \times X \rightarrow[0,1)$ such that for all $x, y \in X$ :

(a) $\lambda(T S x, y) \leq \lambda(x, y)$ and $\lambda(x, S T y) \leq \lambda(x, y)$ $\mu(T S x, y) \leq \mu(x, y)$ and $\mu(x, S T y) \leq \mu(x, y) ;$

(b) $\lambda(x, y)+\mu(x, y)<1$;

(c)

$$
d(S x, T y) \precsim \lambda(x, y) d(x, y)+\mu(x, y) \frac{d(x, S x) d(y, T y)}{1+d(x, y)} .
$$

Then $S$ and T have a unique common fixed point.

By choosing $\mu=0$ in Theorem 2.4, we deduce the following corollary.

Corollary 2.7 Let $(X, d)$ be a complete complex valued metric space and $S, T: X \rightarrow X$. If there exist mappings $\lambda, \gamma: X \times X \rightarrow[0,1)$ such that for all $x, y \in X$ :

(a) $\lambda(T S x, y) \leq \lambda(x, y)$ and $\lambda(x, S T y) \leq \lambda(x, y)$, $\gamma(T S x, y) \leq \gamma(x, y)$ and $\gamma(x, S T y) \leq \gamma(x, y)$

(b) $\lambda(x, y)+\gamma(x, y)<1$;

(c)

$$
d(S x, T y) \precsim \lambda(x, y) d(x, y)+\gamma(x, y) \frac{d(y, S x) d(x, T y)}{1+d(x, y)} .
$$

Then $S$ and T have a unique common fixed point.

The following result is closely related to Corollary 2.5 with $\gamma=0$. The real valued metric space version of this result is an extension of Dass and Gupta's result [7].

Theorem 2.8 Let $(X, d)$ be a complete complex valued metric space and $T: X \rightarrow X$. If there exist mappings $\lambda, \mu: X \times X \rightarrow[0,1)$ such that for all $x, y \in X$ :

(a) $\lambda(T x, y) \leq \lambda(x, y)$ and $\lambda(x, T y) \leq \lambda(x, y)$, $\mu(T x, y) \leq \mu(x, y)$ and $\mu(x, T y) \leq \mu(x, y) ;$

(b) $\lambda(x, y)+\mu(x, y)<1$;

(c)

$$
d(T x, T y) \precsim \lambda(x, y) d(x, y)+\mu(x, y) \frac{d(y, T y)[1+d(x, T x)]}{1+d(x, y)} .
$$

Then T has a unique fixed point. 
Proof Let $x_{0} \in X$ and the sequence $\left\{x_{n}\right\}$ be defined by

$$
x_{n+1}=T x_{n}, \quad \text { where } n=0,1,2, \ldots
$$

We show that $\left\{x_{n}\right\}$ is a Cauchy sequence. From (15), we have

$$
\begin{aligned}
d\left(x_{n+1}, x_{n+2}\right) & =d\left(T x_{n}, T x_{n+1}\right) \\
& \precsim \lambda\left(x_{n}, x_{n+1}\right) d\left(x_{n}, x_{n+1}\right)+\mu\left(x_{n}, x_{n+1}\right) \frac{d\left(x_{n+1}, T x_{n+1}\right)\left[1+d\left(x_{n}, T x_{n}\right)\right]}{1+d\left(x_{n}, x_{n+1}\right)} \\
& =\lambda\left(x_{n}, x_{n+1}\right) d\left(x_{n}, x_{n+1}\right)+\mu\left(x_{n}, x_{n+1}\right) \frac{d\left(x_{n+1}, x_{n+2}\right)\left[1+d\left(x_{n}, x_{n+1}\right)\right]}{1+d\left(x_{n}, x_{n+1}\right)} \\
& =\lambda\left(x_{n}, x_{n+1}\right) d\left(x_{n}, x_{n+1}\right)+\mu\left(x_{n}, x_{n+1}\right) d\left(x_{n+1}, x_{n+2}\right) .
\end{aligned}
$$

It follows from (a) that

$$
\begin{aligned}
d\left(x_{n+1}, x_{n+2}\right) & \precsim \lambda\left(x_{n}, x_{n+1}\right) d\left(x_{n}, x_{n+1}\right)+\mu\left(x_{n}, x_{n+1}\right) d\left(x_{n+1}, x_{n+2}\right) \\
& \precsim \lambda\left(x_{0}, x_{n+1}\right) d\left(x_{n}, x_{n+1}\right)+\mu\left(x_{0}, x_{n+1}\right) d\left(x_{n+1}, x_{n+2}\right) \\
& \precsim \lambda\left(x_{0}, x_{0}\right) d\left(x_{n}, x_{n+1}\right)+\mu\left(x_{0}, x_{0}\right) d\left(x_{n+1}, x_{n+2}\right) .
\end{aligned}
$$

Therefore,

$$
\left|d\left(x_{n+1}, x_{n+2}\right)\right| \leq \lambda\left(x_{0}, x_{0}\right)\left|d\left(x_{n}, x_{n+1}\right)\right|+\mu\left(x_{0}, x_{0}\right)\left|d\left(x_{n+1}, x_{n+2}\right)\right|,
$$

and hence

$$
\left|d\left(x_{n+1}, x_{n+2}\right)\right| \leq \frac{\lambda\left(x_{0}, x_{0}\right)}{1-\mu\left(x_{0}, x_{0}\right)}\left|d\left(x_{n}, x_{n+1}\right)\right|, \quad \text { for all } n=0,1,2, \ldots
$$

Let $h=\frac{\lambda\left(x_{0}, x_{0}\right)}{1-\mu\left(x_{0}, x_{0}\right)}<1$. Then

$$
\left|d\left(x_{n+1}, x_{n+2}\right)\right| \leq h\left|d\left(x_{n}, x_{n+1}\right)\right|, \quad \text { for all } n=0,1,2, \ldots
$$

From Lemma 2.3, we have $\left\{x_{n}\right\}$ is a Cauchy sequence in $(X, d)$. By the completeness of $X$, there exists $z \in X$ such that $x_{n} \rightarrow z$ as $n \rightarrow \infty$. Next, we show that $z$ is a fixed point of $T$. Then

$$
\begin{aligned}
d(z, T z) & \precsim d\left(z, T x_{n}\right)+d\left(T x_{n}, T z\right) \\
& \precsim d\left(z, x_{n+1}\right)+\lambda\left(x_{n}, z\right) d\left(x_{n}, z\right)+\mu\left(x_{n}, z\right) \frac{d(z, T z)\left[1+d\left(x_{n}, T x_{n}\right)\right]}{1+d\left(z, x_{n}\right)} \\
& \precsim d\left(z, x_{n+1}\right)+\lambda\left(x_{0}, z\right) d\left(x_{n}, z\right)+\mu\left(x_{0}, z\right) \frac{d(z, T z)\left[1+d\left(x_{n}, x_{n+1}\right)\right]}{1+d\left(z, x_{n}\right)} .
\end{aligned}
$$

Notice that $\mu\left(x_{0}, z\right) \in[0,1)$. Therefore, we get $d(z, T z) \precsim \mu\left(x_{0}, z\right) d(z, T z)$, that is, $z=T z$. 
Finally, we show the uniqueness. Suppose that there is $z^{\prime \prime} \in X$ such that $z^{*}=T z^{*}$. Then

$$
\begin{aligned}
d\left(z, z^{*}\right) & =d\left(T z, T z^{*}\right) \\
& \precsim \lambda\left(z, z^{*}\right) d\left(z, z^{*}\right)+\mu\left(z, z^{*}\right) \frac{d\left(z^{*}, T z^{*}\right)[1+d(z, T z)]}{1+d\left(z, z^{*}\right)} \\
& =\lambda\left(z, z^{*}\right) d\left(z, z^{*}\right) .
\end{aligned}
$$

Since $\lambda\left(z, z^{*}\right) \in[0,1)$, we have $d\left(z, z^{*}\right)=0$, that is, $z=z^{*}$. This completes the proof.

\section{Deduced results}

\subsection{Sintunavarat and Kumam's results}

We deduce the main result of [4] as follows.

Theorem 3.1 ([4, Theorem 3.1]) Let $(X, d)$ be a complete complex valued metric space and $S, T: X \rightarrow X$. If there exist mappings $\Lambda, \Xi: X \rightarrow[0,1)$ such that for all $x, y \in X:$

(i) $\Lambda(S x) \leq \Lambda(x)$ and $\Xi(S x) \leq \Xi(x)$;

(ii) $\Lambda(T x) \leq \Lambda(x)$ and $\Xi(T x) \leq \Xi(x)$;

(iii) $(\Lambda+\Xi)(x)<1$;

(iv)

$$
d(S x, T y) \precsim \Lambda(x) d(x, y)+\frac{\Xi(x) d(x, S x) d(y, T y)}{1+d(x, y)} .
$$

Then $S$ and $T$ have a unique common fixed point.

Proof Define $\lambda, \mu: X \times X \rightarrow[0,1)$ by

$$
\lambda(x, y)=\Lambda(x) \quad \text { and } \quad \mu(x, y)=\Xi(x), \quad \text { for all } x, y \in X .
$$

Then for all $x, y \in X$,

(a) $\lambda(T S x, y)=\Lambda(T S x) \leq \Lambda(S x) \leq \Lambda(x)=\lambda(x, y)$ and $\lambda(x, S T y)=\Lambda(x)=\lambda(x, y)$; $\mu(T S x, y)=\Xi(T S x) \leq \Xi(S x) \leq \Xi(x)=\mu(x, y)$ and $\mu(x, S T y)=\Xi(x)=\mu(x, y)$;

(b) $\lambda(x, y)+\mu(x, y)=\Lambda(x)+\Xi(x)<1$;

(c)

$$
\begin{aligned}
d(S x, T y) & \precsim \Lambda(x) d(x, y)+\frac{\Xi(x) d(x, S x) d(y, T y)}{1+d(x, y)} \\
& =\lambda(x, y) d(x, y)+\mu(x, y) \frac{d(x, S x) d(y, T y)}{1+d(x, y)} .
\end{aligned}
$$

By Corollary 2.6, $S$ and $T$ have a unique common fixed point.

Remark 1 It is worth mentioning that (i) and (ii) of Theorem 3.1 above can be weakened by the condition

$$
\Lambda(T S x) \leq \Lambda(x) \text { and } \quad \Xi(T S x) \leq \Xi(x), \quad \text { for all } x \in X
$$




\subsection{Rouzkard and Imdad's results}

The following corollary is easily obtained from our Theorem 2.4.

Corollary 3.2 Let $(X, d)$ be a complete complex valued metric space and $S, T: X \rightarrow X$. If there exist mappings $\lambda, \mu, \gamma: X \rightarrow[0,1)$ such that for all $x, y \in X$ :

(a) $\lambda(T S x) \leq \lambda(x), \mu(T S x) \leq \mu(x)$ and $\gamma(T S x) \leq \gamma(x)$;

(b) $\lambda(x)+\mu(x)+\gamma(y)<1$;

(c)

$$
d(S x, T y) \precsim \lambda(x) d(x, y)+\mu(x) \frac{d(x, S x) d(y, T y)}{1+d(x, y)}+\gamma(x) \frac{d(y, S x) d(x, T y)}{1+d(x, y)} .
$$

Then $S$ and $T$ have a unique common fixed point.

Proof Define $\lambda, \mu, \gamma: X \times X \rightarrow[0,1)$ by

$$
\lambda(x, y)=\lambda(x), \quad \mu(x, y)=\mu(x) \quad \text { and } \quad \gamma(x, y)=\gamma(x), \quad \text { for all } x, y \in X .
$$

Then for all $x, y \in X$,

(a) $\lambda(T S x, y)=\lambda(T S x) \leq \lambda(x)=\lambda(x, y)$ and $\lambda(x, S T y)=\lambda(x)=\lambda(x, y)$;

$$
\begin{aligned}
& \mu(T S x, y)=\mu(T S x) \leq \mu(x)=\mu(x, y) \text { and } \mu(x, S T y)=\mu(x)=\mu(x, y) ; \\
& \gamma(T S x, y)=\gamma(T S x) \leq \gamma(x)=\gamma(x, y) \text { and } \gamma(x, S T y)=\gamma(x)=\gamma(x, y) ;
\end{aligned}
$$

(b) $\lambda(x, y)+\mu(x, y)+\gamma(x, y)=\lambda(x)+\mu(x)+\gamma(x)<1$;

(c)

$$
\begin{aligned}
d(S x, T y) & \precsim \lambda(x) d(x, y)+\mu(x) \frac{d(x, S x) d(y, T y)}{1+d(x, y)}+\gamma(x) \frac{d(y, S x) d(x, T y)}{1+d(x, y)} \\
& =\lambda(x, y) d(x, y)+\mu(x, y) \frac{d(x, S x) d(y, T y)}{1+d(x, y)}+\gamma(x, y) \frac{d(y, S x) d(x, T y)}{1+d(x, y)} .
\end{aligned}
$$

By Theorem 2.4, $S$ and $T$ have a unique common fixed point.

Letting $\lambda(\cdot)=\lambda, \mu(\cdot)=\mu$ and $\gamma(\cdot)=\gamma$ in Corollary 3.2 gives the following result proved by Rouzkard and Imdad in [5].

Corollary 3.3 ([5]) If $S$ and $T$ are self-mappings defined on a complete complex valued metric space $(X, d)$ satisfying the condition

$$
d(S x, T y) \precsim \lambda d(x, y)+\mu \frac{d(x, S x) d(y, T y)}{1+d(x, y)}+\gamma \frac{d(y, S x) d(x, T y)}{1+d(x, y)}
$$

for all $x, y \in X$, where $\lambda, \mu, \gamma$ are nonnegative reals with $\lambda+\mu+\gamma<1$, then $S$ and $T$ have a unique common fixed point.

\subsection{Dass and Gupta's results}

Applying the proof of our Theorem 2.8, we can deduce the following result of Dass and Gupta [7] in the context of real valued metric spaces. 
Theorem 3.4 ([7]) Let $(X, d)$ be a real valued metric space. Let $T: X \rightarrow X$ be such that

(i)

$$
d(T x, T y) \leq \lambda d(x, y)+\frac{\mu d(y, T y)[1+d(x, T x)]}{1+d(x, y)}
$$

for all $x, y \in X, \lambda>0, \mu>0, \lambda+\mu<1$, and

(ii) for some $x_{0} \in X$, the sequence of iterates $\left\{T^{n}\left(x_{0}\right)\right\}$ has a subsequence $\left\{T^{n_{k}}\left(x_{0}\right)\right\}$ with

$$
z=\lim _{k \rightarrow \infty} T^{n_{k}}\left(x_{0}\right) \text {. }
$$

Then $z$ is a unique fixed point of $T$.

Proof Define $\lambda, \mu: X \times X \rightarrow[0,1)$ by

$$
\lambda(x, y)=\lambda \quad \text { and } \quad \mu(x, y)=\mu, \quad \text { for all } x, y \in X .
$$

Then the conditions (a), (b) and (c) of Theorem 2.8 are satisfied. Hence, we have $\left\{x_{n}\right\}$ is a Cauchy sequence in $(X, d)$. By (ii), the whole sequence $x_{n} \rightarrow z$ as $n \rightarrow \infty$. It follows again from the proof of Theorem 2.8 that $z$ is a unique fixed point of $T$ as desired.

\section{Competing interests}

The authors declare that they have no competing interests.

\section{Authors' contributions}

All authors contributed equally and significantly to this research work. All authors read and approved the final manuscript.

\section{Acknowledgements}

The authors thank the referee for their comments and suggestions on the manuscript. The first author is supported by Khon Kaen University-Integrated Multidisciplinary Research Cluster (Sciences and Technologies). The research work of the second author was also supported by the Higher Education Research Promotion and National Research University Project of Thailand, Office of the Higher Education Commission, through the Cluster of Research to Enhance the Quality of Basic Education.

Received: 18 July 2012 Accepted: 10 October 2012 Published: 24 October 2012

\section{References}

1. Azam, A, Fisher, B, Khan, M: Common fixed point theorems in complex valued metric spaces. Numer. Funct. Anal. Optim. 32(3), 243-253 (2011)

2. Ding, HS, Kadelburg, Z, Karapinar, E, Radenović, S: Common fixed points of weak contractions in cone metric spaces. Abstr. Appl. Anal. 2012, Article ID 793862 (2012). doi:10.1155/2012/793862

3. Ding, HS, Kadelburg, Z, Nashine, HK: Common fixed point theorems for weakly increasing mappings on ordered orbitally complete metric spaces. Fixed Point Theory Appl. 2012, 85 (2012). doi:10.1186/1687-1812-2012-85

4. Sintunavarat, W, Kumam, P: Generalized common fixed point theorems in complex valued metric spaces and applications. J. Inequal. Appl. 2012, 84 (2012). doi:10.1186/1029-242X-2012-84

5. Rouzkard, F, Imdad, M: Some common fixed point theorems on complex valued metric spaces. Comput. Math. Appl. (2012). doi:10.1016/j.camwa.2012.02.063

6. Bhatt, S, Chaukiyal, S, Dimri, RC: Common fixed point of mappings satisfying rational inequality in complex valued metric space. Int. J. Pure Appl. Math. 73(2), 159-164 (2011)

7. Dass, BK, Gupta, S: An extension of Banach contraction principle through rational expression. Indian J. Pure Appl. Math. 6(12), 1455-1458 (1975)

doi:10.1186/1687-1812-2012-189

Cite this article as: Sitthikul and Saejung: Some fixed point theorems in complex valued metric spaces. Fixed Point Theory and Applications 2012 2012:189. 